\title{
Error Reduction based Demand Forecasting: An Appraisal of Kerala Power System
}

\author{
K. Pramelakumari \\ Associate Professor \\ Electrical and Electronics \\ Engineering \\ Government Engineering College, \\ Thrissur
}

\author{
V. P. Jagathy Raj, PhD \\ Professor \\ School of Management Studies \\ Cochin University of Science and \\ Technology, Kochi-22
}

\author{
P. S. Sreejith, PhD \\ Professor and Dean \\ Faculty of Engineering \\ Cochin University of Science and \\ Technology, Kochi-22
}

\begin{abstract}
Demand forecasting is a procedure for identifying the electrical demand that can be expected from a specified number of consumers in a specified period. Electrical demand and electrical supply system could be in terms of average system demand, maximum system demand, and load demand in MW or energy demand in MWhr. Demand forecasting is usually undertaken with the prediction of hourly, daily, weekly and annually of the system demand and peak demand. Categorized form of load forecasting are short-term, longterm and medium-term which takes a few hours ahead of few weeks, one week to one year and five years to twenty years etc. This paper describes the demand forecasting of Kerala power system by using two different methods and a comparative analysis on its impact on the accuracy of load forecasting. This paper also present an error reduction based demand forecasting of Kerala power system. The performance evaluation parameters MAPE, MSE, RMSE, MAE/MAD and percentage error have been used for testing this proposed forecasting models with error reduction strategies.
\end{abstract}

\section{Keywords}

Demand forecasting, optimization, neural network and time series.

\section{INTRODUCTION}

For a power system, the peak-cliff and off-peak troughs are significant and the forecast should be able to identify these factors with more accuracy rather than minimizing the error in the energy content. The share of electricity in total final energy consumption is expected to rise to a marked extent. The issues of environmental pollution and the shortage on the availability of fossil fuel tend to replace several conventional applications with electrical power. The demand models relate variation of a system based on maximum demand with time that can be specified as day, month, quarter or year. The demand models are autonomous models and conditional models. Autonomous models which relate future growth of electricity demand of a system based on its past growth, whereas the conditional models attempt to relate electricity demand growth to other variables like economic indicators. It requires details on loads, weather patterns, times of cultural activities and ceremonies [1].

A number of techniques has been conventionally used for electric energy demand forecasting. Most forecasting methods use statistical techniques or artificial intelligence algorithm like regression, neural networks, fuzzy logic and expert systems. Various regression models such as Auto Regressive Moving Average (ARMA), Auto Regressive Integrated Moving Average (ARIMA), Auto Regressive Moving Average with Exogenous variables (ARMAX), Auto
Regressive Integrated Moving Average with Exogenous variables (ARIMAX), and time series have been developed for short-term forecasting. These are the classical time series methods. [2].

The forecast error in load prediction results in increased operating costs. Under-prediction of load results is a failure to provide necessary reserves which translates into higher costs due to the use of expensive peak hour units. Over-prediction of load results in an unnecessary increase of reserves and hence increases the operating cost.

The two different techniques concentrated here are artificial neural network method and the time series approach. The rest of the paper is organized as follows: Section 2 introduces a brief sketch of Kerala Power System. Section 3 analyses the short-term demand forecast modelling by artificial neural network. Section 4 reviews the modelling by time series. Section 5 shows the comparison on evaluation of forecasted errors of neural network and time series. Section 6 discusses the accuracy of forecasted values and the paper is concluded in section 7 .

\section{KERALA POWER SYSTEM: A BRIEF SKETCH}

The Kerala power system is different from other States in many respects. Electric current produced from hydroelectric process costs only Rs. 2 per unit, while current obtained from thermal method costs more than Rs.10 per unit. Kerala had achieved a status of a power surplus state till 1987. During this period of 1969-1987, Kerala was selling power to the neighboring States at very cheap rates. The State also resolved to encourage the setting up of power intensive industrial units. In spite of this, Kerala faced a reversal of the situation with power shortages of varying magnitudes, depending on the intensity and vagaries of monsoon. Due to drastic drop in rainfall in the south monsoon season, there had been a serious supply shortage for power in the state. This had forced to introduce one hour load shedding of households and power cut of industries. [3]

The Kerala power map is having its own characteristics. It is different from other States in many respects. Vagaries of monsoon, high dependence on costlier power, high peak-off peak ratio, unfavorable consumer mix(adverse), High-tension - Low-tension (HT-LT) ratio, lack of energy resources, transmission constraints of import power from one side of the state to another and exposure to global oil prices are some of the significant factors of this system. The cost of power procurement alone works out at present $70 \%$ of the total revenue requirement of Kerala State Electricity Board (KSEB) depending on the availability of monsoon. These aspects necessitate optimization in the management of power 
system. The cost of power depends on source of power. Hence any optimization strategy in the management of power system involves scheduling of power from various sources. As the scheduling involves many technical and commercial considerations and constraints, the efficiency in scheduling depends on the accuracy of load forecast. Thus the load forecasting for Kerala power system has become an important mechanism for planning and operation.

\section{SHORT TERM DEMAND FORECAST MODELING BY ARTIFICIAL NEURAL NETWORK}

The load forecasting helps an electric utility company to make significant decision on purchasing of power, load switching, schedule the power exchange from Central Generating Stations, control the number of running generating units and schedule the device maintenance plan. The short-term load forecasting has an essential part in power system planning, operation and control [4], [5].

Artificial Neural Network (ANN) contains the structure and behavior of human brain. It is analogous to the human nervous system. The neural network's structure consists of several layers of processing units called neurons. Artificial neural network is an information processing paradigm that is modeled to the way of biological nervous system works, such as the brain's information etc. Multi layered back propagation algorithm is used in this work. The back propagation neural network model consists of an input layer, one or more hidden layers and an output layer. In addition to the processing neuron, there is a bias neuron connected to each processing unit in the hidden and output layers. The number of hidden layers and neurons within each layer can vary depending on the size and nature of data set [6]. The anti- propagation error is carried out by contrasting the actual output with desired output. Each weight is revised and back propagated layer by layer from output layer to hidden layer and input layer. This process will be continued until the output error of network is reduced to an acceptable level [7].

ANN based models for load forecasting has been used most successfully. The model can be denoted by the following equation:

$P_{t+1}=\left(t, p_{t}, p_{t-1} \ldots . P_{t-n}, w_{t}, w_{t-1} \ldots w_{t-r}, \hat{w}_{t+1}\right)+\varepsilon_{t+1}$

$\mathrm{t}=$ time of the day, $\mathrm{Pt}=$ load at time $\mathrm{t}$, wt $=$ weather factors observed at time t. $1=$ time lead of the forecast, $\hat{w}_{t+1}=$ weather forecast for time $\mathrm{t}+1, \quad \varepsilon_{t+1}=\mathrm{a}$ random load component.

For short term demand forecasting with maximum time leads, the weather variables can be neglected due to relatively large time constant of the load-weather relationship. Then the equation (1) can be re-written as:

$P_{t+1}=f\left(t, p_{t}, p_{t-1} \ldots . P_{t-n}\right)+\varepsilon_{t+1}$

Using this equation the load forecast $\mathrm{pt}+\mathrm{l}$ is calculated directly by the trained neural network based on the time and loads from the recent past [8].

Figure 1 shows the actual and predicted load curves for one day ahead (for April 9, 2015) using previous day data of April 8,2015 . It is clear from the results that the forecasted load patterns are very much similar to the actual load by artificial neural network method.
Actual Vs Predicted 1 Day ahead

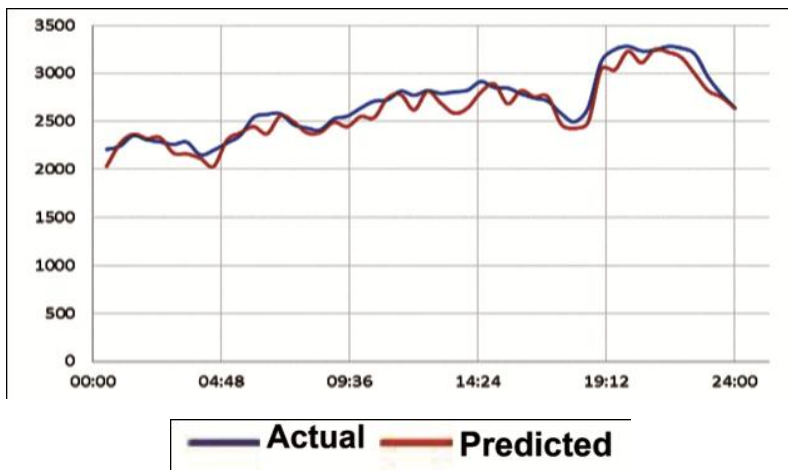

Fig 1: Actual and predicted load curves for one day ahead

Figure 2shows the error between the actual and predicted load curve for one day ahead using the previous day data.

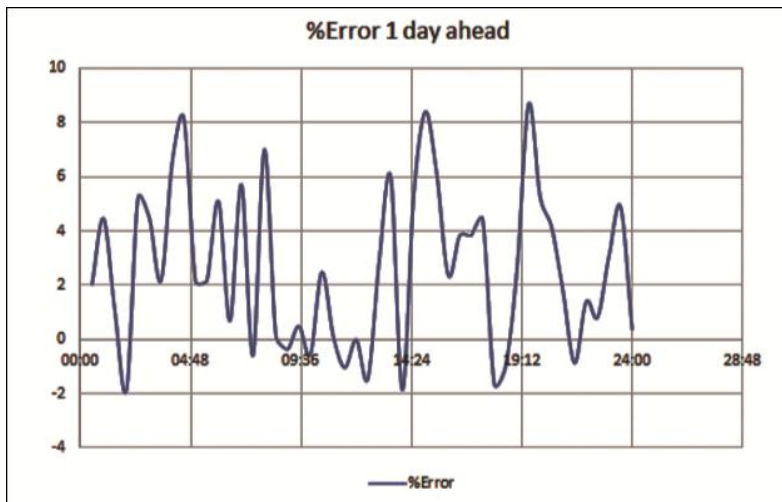

Fig 2: Percentage error curve for one day ahead

Figure 3 shows the actual and predicted load curves for one week ahead for June 12,2015 using previous data for June 5th 2015 data training.

It is evident from the resulting curves, the actual and predicted values are very much similar to one week ahead.

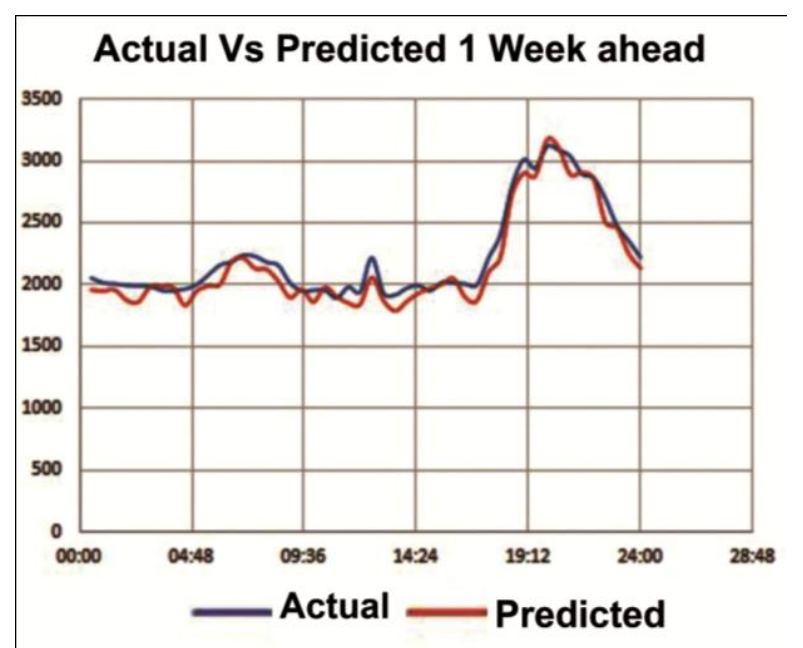

Fig 3: Actual and predicted load curves for one week ahead

Figure 4 shows the percentage error curve corresponding to the actual and forecasted load for one week ahead. 


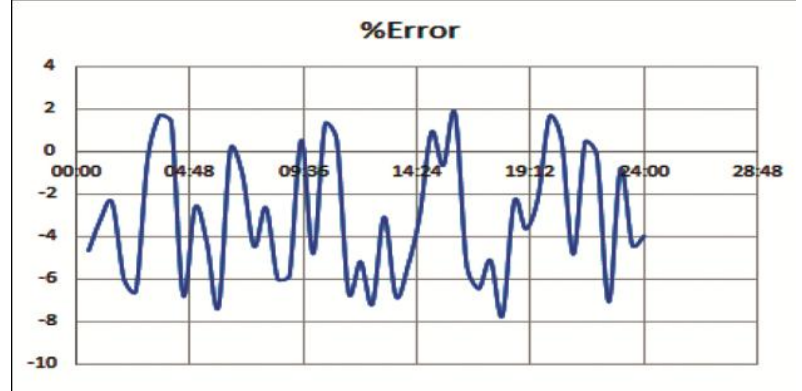

Fig 4: Percentage error curve for one week ahead

\section{SHORT TERM LOAD FORECAST MODELING BY TIME SERIES METHOD}

In time series analysis, the partial autocorrelation function (PACF) gives the partial correlation of a time series with its own lagged values, controlling for the values of the time series at all shorter lags. It contrasts with the auto-correlation function, which does not control for other lags.

The idea of the time series signal is based on a load pattern with known seasonal, weekly and daily periodicities. These periodicities give a rough prediction of the load at the given season, day of the week and time of the day [9]. The difference between the prediction and actual load can be considered as a stochastic process. The techniques used for this random signal include the Kalman filtering, Box-Jenkins method and Auto Regressive Moving Average models. Time series methods are based on the assumption that the data have an internal structure, such as auto-correlation, trend or seasonal variation. Time series forecasting methods detect and explore such as a structure. Among the classical time series methods, such as ARMA, ARIMA ARMAX and ARIMAX. ARMA and ARIMA use the time and load as the only input parameters [10]. Since the load depends on weather and among the classical series model, time series model, which is built upon fitting a time series to the original data. It takes the form as:

$Y_{t}=\alpha+\beta x_{t}+U_{t}$

$Y_{t}=F_{(t)}+U_{t}$

$Y_{t}$ is the demand at time $t, F(t)$ is a function of time that defines the peculiarity of the function used for the time series model and $\mathrm{u}_{\mathrm{t}}$ is a constant that depends on a nature of historical data as well as the statistical process used. This function plays an important role in data analyses aimed at identifying the extent of the lag in an autoregressive model. The use of this function was introduced as part of the BoxJenkins approach to time series modelling, where by plotting the partial auto correlative functions one could determine the appropriate lags $\mathrm{p}$ in an AR (p) model or in an extended ARIMA ( $\mathrm{p}, \mathrm{d}, \mathrm{q}$ ) model. $\mathrm{t}, \mathrm{t}-1, \mathrm{t}-2 \ldots \mathrm{t}-\mathrm{k}$ is the previous value or lagged value. $t, t+1, t+2 \ldots t+k$ is the future value called as lead value. Time series is a collection of random variables arranged in order of time. $\mathrm{Yt}$ is stochastic variable. $\mathrm{Y}_{\mathrm{t}}, \mathrm{Y}_{\mathrm{t}-1}$, $\mathrm{Y}_{\mathrm{t}-2 \ldots} \ldots \mathrm{Y}_{\mathrm{t}-\mathrm{k}}$ is the lagged stochastic variable. Auto Regressive (AR) process is the form as:

$Y_{t}=\alpha+\beta y_{t-1}+U_{t}$

$\mathrm{Y}_{\mathrm{t}-1}$ is one lag. Therefore auto regressive of order

1. i.e., $\operatorname{AR}(1)$
$Y_{t}=\alpha+\beta_{1} y_{t-1}+\beta_{2} y_{t-2}+u_{t}$

Auto regressive of order 2

$Y_{t}=\alpha+\beta_{1} y_{t-1}+\beta_{2} y_{t-2}+u_{t}+\ldots .+\beta_{p} Y_{t-p}+u_{t}(7)$

Auto regressive of order $\mathrm{p}$

$\beta$ is the Auto-regression coefficient.

A moving average (MA) process is the current value of error change and past values or past observations of system demand or successive realizations of random variables.

$$
\begin{aligned}
& Y_{t}=u_{t}+\gamma_{1} u_{t-1} \ldots \ldots . M A(1) \\
& Y_{t}=u_{t}+\gamma_{2} u_{t-2} \ldots . . M A(2) \\
& Y_{t}=u_{t}+\gamma_{1} u_{t-1}+\gamma_{2} u_{t-2}+\ldots .+\gamma_{q} u_{t-q} \ldots \ldots . M A(q)
\end{aligned}
$$

A combination of Auto Regressive model and moving average model known as Box- Jenkins model or ARMA model.

The model of ARMA is

$$
Y_{t}=\alpha+\phi y_{t-1}+\varepsilon_{t}+\theta \varepsilon_{t-1} \operatorname{ARMA}(1,1)
$$

For modeling ARMA, a trial and error method is to be adopted. $\phi$ is the route and $\varepsilon$ is a random variable. ARIMA model is the Box- Jenkins model with integrated process. The form of ARIMA model is as follows:

$Y_{t}=y_{t-1}+\varepsilon_{t . I}$

This is a non-stationary series.

$Y_{t} \sim \mathrm{I}(1)$

$\Delta_{y t} \sim \mathrm{I}(0)$

$Y_{t} \sim \mathrm{I}(2)-2$ unit route i.e., two different roots.

$\Delta_{2 y t} \sim \mathrm{I}(0)$ differentiate two times to make it stationary.

$Y_{t} \sim \mathrm{I}(\mathrm{d})$ ARIMA $(1, \mathrm{~d}, 1)$

$\Delta_{d y t} \sim \mathrm{I}(0)$. This is a stationary series. $\operatorname{ARIMA}(1,1)$ or $\mathrm{yt} \sim \mathrm{I}$ (d), ARIMA (p, d, q).

Figure 5 shows the sequence plot of the hourly demand data for one year from April 18, 2014 to March 31, 2015. An increasing trend is seen in the plot and is not stationary.

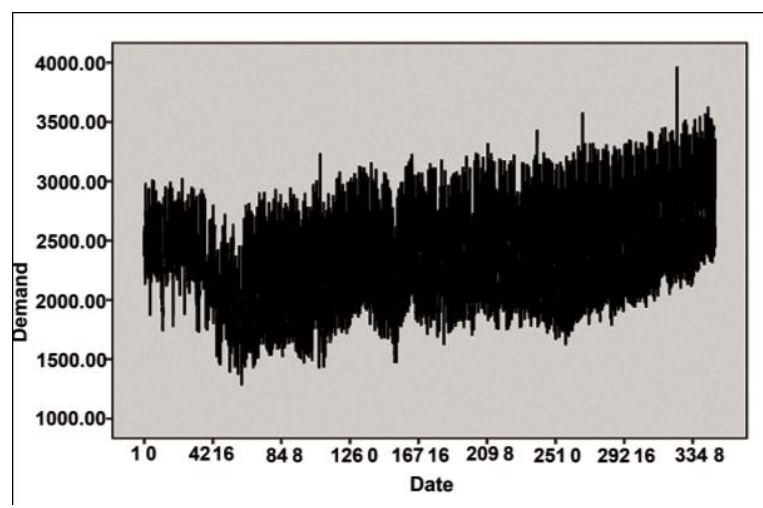

Fig 5: Sequence Plot for Actual Data

To make the data stationary, a first difference transformation was made and the plot is optioned as follows. 


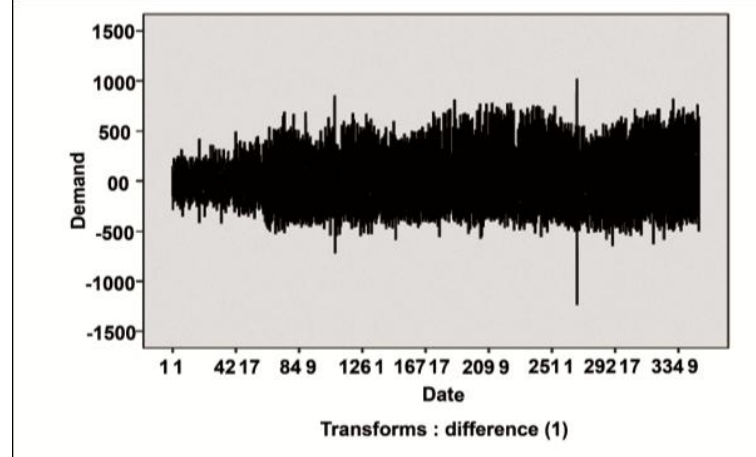

Fig 6: Sequence Plot for Transformed Data with First Difference

Figure 6 shows the sequence plot of the transformed data for one year from April 18, 2014 to March 31, 2015. Now the data is stationary. Fixed the time series model ARIMA (1, 1, 2) $(1,1,1)$ for the above data, then the time plot of the model with actual observations and forecasted observations is obtained as shown in figure 7 .

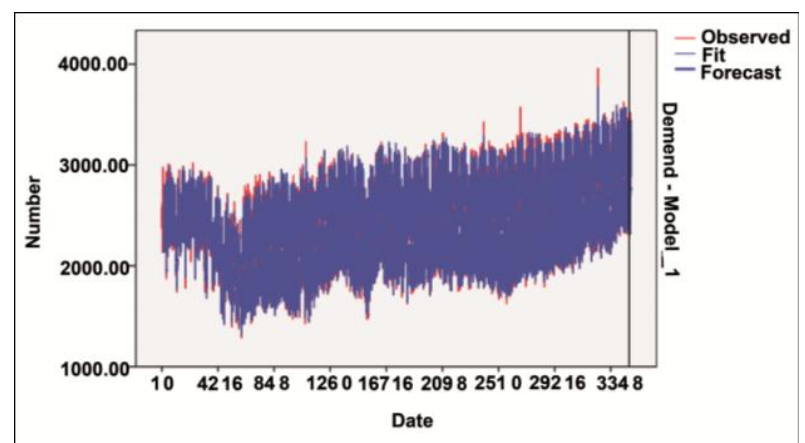

Fig 7: Sequence Plot of Actual and Forecasted Values

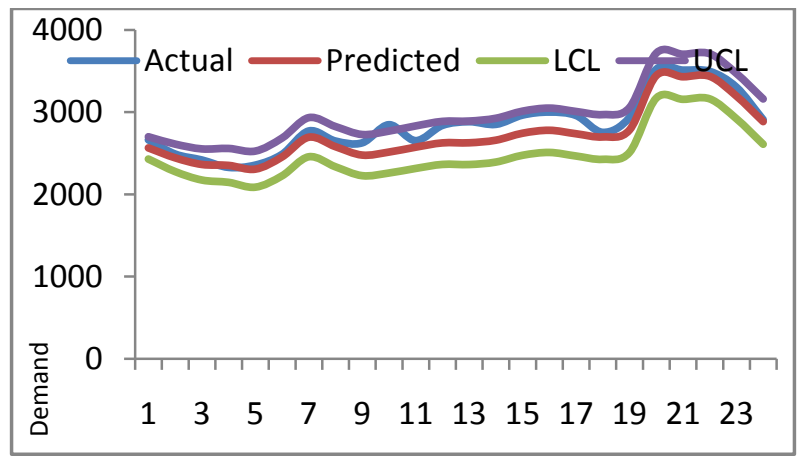

Fig 8: Predicted Observations and the Control Limits (Insample Prediction)

Now for checking the effectiveness of the proposed model in forecasting future values, an in-sample forecasting of last 21 days was done. For this, the data up to March 30, 2015, were taken and forecast the 24 hour demand observations for March 31, 2015 by using the model and also developed and compared the values with actual data. From the Figure 8 and Table 1, it can be inferred that the forecasted and observed values are almost same. That is the model is effective in forecasting.
Table 1: Predicted Observations and the Control Limits (In-sample Prediction)

\begin{tabular}{|c|c|c|c|c|c|}
\hline & Actual & Predicted & LCL & UCL & $\begin{array}{l}\text { \% } \\
\text { Error }\end{array}$ \\
\hline Hour1 & 2664.58 & 2566.39 & 2431.17 & 2701.61 & 3.68 \\
\hline Hour2 & 2491.13 & 2445.49 & 2278.72 & 2612.27 & 1.83 \\
\hline Hour3 & 2418.64 & 2364.85 & 2175.69 & 2554.00 & 2.22 \\
\hline Hour4 & 2328.47 & 2353.55 & 2147.54 & 2559.56 & 1.07 \\
\hline Hour5 & 2356.22 & 2308.05 & 2088.94 & 2527.16 & 2.04 \\
\hline Hour6 & 2487.24 & 2456.05 & 2226.56 & 2685.55 & 1.25 \\
\hline Hour7 & 2772.16 & 2696.14 & 2458.31 & 2933.98 & 2.74 \\
\hline Hour8 & 2649.55 & 2581.07 & 2336.48 & 2825.66 & 2.58 \\
\hline Hour9 & 2627.60 & 2478.87 & 2228.76 & 2728.98 & 5.66 \\
\hline $\begin{array}{l}\text { Hour1 } \\
0\end{array}$ & 2848.27 & 2515.77 & 2261.13 & 2770.41 & 11.67 \\
\hline $\begin{array}{l}\text { Hour1 } \\
1\end{array}$ & 2654.17 & 2575.30 & 2316.93 & 2833.67 & 2.97 \\
\hline $\begin{array}{l}\text { Hour1 } \\
2\end{array}$ & 2840.15 & 2627.13 & 2365.67 & 2888.58 & 7.50 \\
\hline $\begin{array}{l}\text { Hour1 } \\
3\end{array}$ & 2885.05 & 2628.38 & 2364.37 & 2892.40 & 8.89 \\
\hline $\begin{array}{l}\text { Hour1 } \\
4\end{array}$ & 2852.69 & 2660.24 & 2394.10 & 2926.38 & 6.74 \\
\hline $\begin{array}{l}\text { Hour1 } \\
5\end{array}$ & 2965.93 & 2744.97 & 2477.06 & 3012.88 & 7.44 \\
\hline $\begin{array}{l}\text { Hour1 } \\
6\end{array}$ & 3002.40 & 2780.56 & 2511.17 & 3049.95 & 7.38 \\
\hline $\begin{array}{l}\text { Hour1 } \\
7\end{array}$ & 2960.90 & 2738.38 & 2467.75 & 3009.00 & 7.51 \\
\hline $\begin{array}{l}\text { Hour1 } \\
8\end{array}$ & 2755.36 & 2699.33 & 2427.68 & 2970.99 & 2.03 \\
\hline $\begin{array}{l}\text { Hour1 } \\
9\end{array}$ & 2937.37 & 2784.24 & 2511.72 & 3056.76 & 5.21 \\
\hline $\begin{array}{l}\text { Hour2 } \\
0\end{array}$ & 3524.42 & 3445.31 & 3172.06 & 3718.56 & 2.24 \\
\hline $\begin{array}{l}\text { Hour2 } \\
1\end{array}$ & 3513.46 & 3431.63 & 3157.77 & 3705.49 & 2.32 \\
\hline $\begin{array}{l}\text { Hour2 } \\
2\end{array}$ & 3498.45 & 3437.24 & 3162.86 & 3711.61 & 1.74 \\
\hline $\begin{array}{l}\text { Hour2 } \\
3\end{array}$ & 3305.77 & 3196.51 & 2921.71 & 3471.32 & 3.30 \\
\hline $\begin{array}{l}\text { Hour2 } \\
4\end{array}$ & 2909.59 & 2886.31 & 2611.14 & 3161.48 & 0.80 \\
\hline
\end{tabular}

Now the remaining data points were augmented to analyze the complete sample. Analysis of the complete data yields the same model as before. In all model diagnostic checking procedures are done. Thus, by making use of above model up to March 31, 2015, daily absenteeism for the next 24 hours load were forecasted, and the values are obtained and are given in table 1 . 


\section{EVALUATION OF FORECASTED ERRORS OF NEURAL NETWORK AND TIME SERIES: A COMPARISON}

If statistical models are used for forecasting, error can be evaluated statistically. Forecast errors have two components, model error and assumption error [11]. These errors can be assessed as soon as the forecast is completed and before the actual data is known. In calculating a forecast error the variance is calculated first and to obtain forecast error by taking the square root. Model error is inherent in the model itself. Its magnitude depends on the model's variance, variance of the estimated coefficients and the changes in the values of independent variables. A good forecasting model should have small variance. The assessment of the artificial neural network and time series models prediction performance was done by quantifying the prediction obtained on an independent data set. The mean absolute error ( MAE), the mean absolute percentage error (MAPE), and route mean square error (RMSE) were used to represent the performance of the above two prediction models.

$M A E=M A D=\frac{1}{n} \times \sum_{t=1}^{n} e_{t}$ where, $e_{t}=\left|L_{r t}-L_{f t}\right|$

Therefore MAE $=\frac{1}{n} \times \sum_{t=1}^{n}\left|L_{r t}-L_{f t}\right|$

$L_{f t}$ is the forecasted load for a period t, $L_{r t}$ is the actual load of period $\mathrm{t}$.

$\mathrm{n}$ is the number of total data or number of period of evaluation and et is the absolute error.

$\mathrm{MSE}=\frac{1}{n} \sum_{t=1}^{n} e_{t^{2}}=\frac{1}{n} \sum_{t=1}^{n}\left|L_{r t}-L_{f t}\right|^{2}$

Root Mean Squared error,

$\mathrm{RMSE}=\sqrt{M S E}=\left[\frac{1}{n} \sum_{t=1}^{n}\left|L_{r t}-L_{f t}\right|^{2}\right]^{1 / 2}$

Mean absolute percentage error,

$$
\begin{aligned}
\text { MAPE } & =\frac{1}{n} \sum_{t=1}^{n}\left|\frac{e_{t}}{L_{r t}}\right| \times 100 \\
& =\frac{1}{n} \sum_{t=1}^{n}\left|\frac{L_{r t}-L_{f t}}{L_{r t}}\right| \times 100
\end{aligned}
$$

From the two methods (neural network and time series) percentage error has been calculated and also MAPE, MPE, MSE and MAE are calculated and compared for each method. From the comparison the percentage error of the neural network method shows lower than the time series method.

\section{FORECAST ACCURACY}

Accuracy is a relative term i.e., a $1 \%$ error may be too large for one case, where as a 5\% error is acceptable in another. In the case of Kerala power system, actual load is less than the forecasted load in most of the cases. Mean absolute percentage error (MAPE) is another measure of prediction accuracy in which find the absolute percentage error for each forecast and computing the average of these values. The advantage of MAPE is that it allows comparison among different series which are not possible with mean square error (MSE). The MAPE is varied from 1.63 to 13.053 for the year 2014 to 2016. For reducing the forecast error on an annual basis is to develop hourly or weekly or monthly or one dayahead models for forecasting to take advantage of the least squares estimation property. All regression and econometric models are estimated with the least squares method. The positive and negative residuals of a least squares model add up to zero. If the model fits the data well, it can be expected that monthly or quarterly forecast errors for a given year will sum up to a small number. But monthly data often will contain a number of constraints. These constraints can cause the model to have large errors affecting forecast accuracy. A compromise is to be used for quarterly data to smooth out those constraints [12].

When prediction is carried out over several periods, a series of error values are obtained for each time period [13],[14]. Mean square error (MSE) method is a better approach for examining the accuracy of prediction used in this study. MSE is computed first squaring the individual errors, which give all positive values and then taking the average value of all data points. This approach gives more importance to large errors in the mean squared error computation than small errors. If absolute errors are used in place of the absolute percentage errors, the mean absolute deviation (MAD) is obtained. Another measure of prediction error is the mean percentage error (MPE). The calculated values of MAD, MSE, mean absolute percentage error (MAPE) and route mean square error (RMSE) are shown in Table 2.

Table 2. Forecasting errors by different approaches for 2014-2016

\begin{tabular}{|l|l|l|l|l|l|}
\hline \multicolumn{6}{|l|}{ By NN } \\
\hline Year & MAE/MAD & MPE & MAPE & MSE & RMSE \\
\hline 2014 & 22.5 & 0.35409 & 1.6307 & 1129.833 & 33.61298 \\
\hline 2015 & 57.508 & 0.8418 & 3.2870 & 6889.49 & 83.002951 \\
\hline 2016 & 52.254 & 0.72681 & 3.1255 & 8962.77 & 94.6719 \\
\hline By Time Series & & & & \\
\hline 2014 & 107.287 & 2.5878 & 3.8645 & 20088.83 & 141.73507 \\
\hline 2015 & 142.295 & 3.428 & 5.8901 & 29050.27 & 170.44139 \\
\hline 2016 & 139.162 & 8.69629 & 6.2976 & 33125.23 & 182.003379 \\
\hline
\end{tabular}

\section{CONCLUSION}

A case study for the proposed two methods was carried out for forecasting monthly and weekly electric load using the data obtained from Kerala State Electricity Board. The results were obtained for three years from 2014 to 2016. The comparison of forecasted load and actual load are shown in figures. Besides, the experimental models give better forecasting results with the overall average percentage error of $6.58 \%$. Generally $10 \%$ forecasting error is said to be acceptable for long term load forecasting. Table 2 presents the actual forecast error statistics for each model across the five error measures. The MAE statistics indicates that ANN model provides the most accurate forecasts, the time series model ranks a close second. The RMSE statistics also indicate that ANN model provides the most accurate forecasts. The MAPE statistics give a relative indication of overall forecasting performance.

In addition to the conventional forecasts of hourly consolidated loads for the next several days, the instantaneous 
load predictions for the next several hours are also required to operate the power system reliably and economically. This study used a practical method for comparison of two methods like time series and neural network models to estimate shortterm load forecasting and the prediction accuracy has been found. A neural network depends more on training data and the learning algorithm. The forecasted values when compared with actual values are found to be within a MAPE range of $1.63 \%$ to $8.63 \%$, which is acceptable for the system based on large scale. It was observed that the forecasted load by ANN model is very much similar to the actual load with an accuracy of ANN model. The performance evaluation parameters MAPE, MSE, RMSE, MAE/MAD and \% error have used for testing this proposed forecasting models with error reduction strategies. The values of these parameters are very low for ANN model as compared to the time series. It is clear from the test results that the artificial neural network technique works more effectively when compared to the conventional time series technique.

\section{REFERENCES}

[1] A.Obok Opok, G.O. Anderson and K.M. Yanev, "Electricity Demand Forecasting Towards Vision 2016 for Botswana Using Decomposition Time Serial Model," International Journal of Power and Energy Systems, Vol.28,No.2, pp.229-237, 2008.

[2] P. R. J. Campbell and K. Adamson, "Methodologies for Load Forecasting," $3^{\text {rd }}$ International IEEE Conference Intelligent Systems, September, pp. 800-806, 2006.

[3] Economic Review, 1997, State Planning Board, Government of Kerala.

[4] D. C. Park, M. El-Sharkawi, R. Marks, A. Atlas and M. Damborg, "Electric Load Forecasting Using an Artificial Neural Network." IEEE Trans. on Power Systems, Vol. 6, No. 2, pp. 442-449, May 1991.

[5] Mohan B. Tasre, Vilas N. Ghate and Prashant P. Bedkar, "Hourly Load Forecasting Using Artificial Neural Network for a Small Area,'IEEE International Conference on Advances in Engineering, Science and Management, pp.379-385, 2012.
[6] V. Shrivastava, R. B. Misra, "A Novel Approach of Input Variable Selection for ANN Based Load Forecasting," Power System Technology and IEEE Power India Conference, p. 5, 2008.

[7] Amit Jain, IEEE and B. Satish, "Short-Term Load Forecasting by Clustering Technique Based on Daily Average and Peak Loads," IEEE Conference,p.6, 2009.

[8] W. Charytoniuk and Mo-Shing Chen, "Short-Term Load Forecasting Using Artificial Neural Networks," IEEE Transactions on Power Systems, Vol.15, No. 1, pp. 263$268,2000$.

[9] Carolina Gar cia-Ascanio, Carlos Mate, "Electric Power Demand Forecasting Using Interval Time Series: A Comparison between VAR and iMLP," Energy Policy, Vol.38, pp.715-725, 2010

[10] I. Aquino, C. Perez, J.K. Chavez and S. Oporto, "Daily Load Forecasting Using Quick Propagation Neural Network with a Special Holiday Encoding," Proceedings of International Joint Conference on Neural Networks, Orlando, Florida, USA, August 12-17, 2007.

[11] B. J. Chen, M.W. Chang and C. Jen Lin, "Load Forecasting Using Support Vector Machines: A Study on EUNITE Competition 2001," Electrical Power and Energy System, Vol. 26 No.2,pp 1-7.

[12] George C. S. Wang, “ Forecasting Practices in Electric and Gas Utility Companies," The Journal of Business Forecasting Methods \& Systems, Spring, pp.11-13, 2004.

[13] N.Hodzic, T.Konjic and V.Miranda, "Artificial Neural Networks Applied to Short Term Load Diagram Prediction," 8th Seminar on Neural Network Applications in Electrical Engineering, pp. 219-223, 2006.

[14] K. Y. Lee, Y. T. Cha and J. H. Park,"Short-term Load Forecasting using an Artificial Network,"IEEE Trans. on Power Systems, Vol. 7, No.1, pp. 124-132, Feb. 1992. 